\title{
FILTRATIONS AND ASYMPTOTIC AUTOMORPHISMS ON NILPOTENT LIE GROUPS
}

\author{
ROE GOODMAN
}

\section{Introduction}

Let $g$ be a real, nilpotent finite-dimensional Lie algebra. In this paper we compare the following three Lie group structures on $g$ :

(i) the additive group structure $x+y$ of the vector space $g$,

(ii) the multiplicative group structure $x y$ obtained from the Lie bracket $[x, y]$ by the Campbell-Hausdorff formula,

(iii) a multiplicative group structure $x \circ y$ obtained by taking a Lie algebra filtration of $g$, mapping $g$ into the associated graded algebra to obtain a new Lie bracket $\{x, y\}$, and then substituting this new bracket operation into the Campbell-Hausdorff formula.

These Lie group structures have much in common. The operation of inversion is $x \rightarrow-x$ in all cases, and Haar measure is Euclidean measure $d x$. Near the identity element the group operations coincide "to first order," of course. The basic result of this paper is that these three operations are also close at infinity, provided the measurement of size is made via a nonisotropic gauge rather than by the Euclidean norm.

The chief tools we use are one-parameter groups of dilations $\left\{\delta_{r}\right\}_{r>0}$ and gauges $|x|$ which are homogeneous relative to the dilations : $\left|\delta_{r} x\right|=r|x|$ (definitions in $\S 3$ ). The simplest situation occurs when $\delta_{r}$ can be taken as an automorphism of the Lie algebra structure. In this case Knapp-Stein [7] (where a suitable power of a homogeneous gauge is called a "norm function") and Korányi-Vági [6] have used gauges and dilations to extend the CalderonZygmund singular integral operator theory from the additive group of $g$ to the noncommutative group $G$.

In general, the automorphism group of a nilpotent Lie algebra need not contain any dilations [4]. Equivalently, $\mathfrak{g}$ may not admit any Lie algebra gradations. However, g always admits a separating Lie algebra filtration (e.g, the descending central series), and the graded Lie algebra structure associated to the filtration does admit dilating automorphisms $\left\{\delta_{r}\right\}$. In $\S \S 4-6$ we prove that the graded multiplication $x \circ y$ is obtained from the multiplication $x y$ by taking the limit as $r \rightarrow \infty$ of $\delta_{1 / r}\left(\delta_{r} x \delta_{r} y\right)$. We estimate the differences $\mid x y-$

Communicated by J. J. Kohn, April 17, 1975. Research supported in part by NSF Grant GP33567. 
$x \circ y \mid$ and $|x+y-x y|$, measured by a $\delta_{r}$-homogeneous gauge. In particular, $\delta_{r}$, which is an exact automorphism of the group structures $x+y$ and $x \circ y$, is shown to be an asymptotic automorphism of $x y$ (as $|x|+|y| \rightarrow \infty)$. Using these estimates, we prove an estimate for $|f(x y)-f(x)|$, where $f$ is a smooth homogeneous function. (In the graded case this estimate was obtained by Korányi and Vagi [6].)

In $\S 7$ we reverse the order of construction. Starting with an arbitrary dilation group $\delta_{r}$ on Euclidean space and the infinite-dimensional Lie algebra $\mathscr{L}$ of vector fields with polynomial coefficients, we use the induced action of $\delta_{r}$ on $\mathscr{L}$ to obtain a finite-dimensional nilpotent Lie subalgebra $\mathfrak{n}$ of $\mathscr{L}$. The dilations $\delta_{r}$ define a gradation and filtration on $\mathfrak{n}$. Using this, we show that a construction given by Auslander et al [1] in terms of canonical coordinates on a nilpotent group $G$ amounts to embedding $g$ as a filtered subalgebra of $\mathfrak{n}$. The "modified" multiplication on $G$ defined in [1] is then the graded multiplication $x \circ y$ determined by the filtration on $g$ induced by this embedding. (This embedding is essentially an analytic version of Birkhoff's embedding of $g$ as a subalgebra of the upper trianglar matrices [2].)

In a sequel to this paper we use the results of $\S 5$ to study certain translationinvariant spaces of entire functions on a complex nilpotent group $G_{c}$. The present estimates allow us to obtain a "best possible" result concerning the analytic continuation of Banach space representations of a real form $G$ to holomorphic representations of $G_{\boldsymbol{c}}$.

\section{Notation and preliminaries}

As usual $\boldsymbol{N}, \boldsymbol{R}$, and $\boldsymbol{C}$ denote the sets of nonnegative integers, real numbers, and complex numbers, respectively. For $\lambda \in C,|\lambda|$ denotes the usual absolute value. (The same symbol will be used for gauge functions, but it will be clear from the context which is meant.)

$\mathrm{g}$ will be a finite-dimensional nilpotent Lie algebra over $R$, and $\|x\|$ for $x \in \mathfrak{g}$ will be a Euclidean norm defined relative to some basis for g. $G$ will be the simply-connected group with Lie algebra $\mathfrak{g}$. We will identify $G$ with $\mathfrak{g}$ as an analytic manifold via the exponential map. The group multiplication on $G$ is then defined via the Campbell-Hausdorff formula [3]. The group operations have the form

$$
\begin{gathered}
x^{-1}=-x, \\
x y=x+y+\tau(x, y),
\end{gathered}
$$

where $\tau$ is a polynomial function of $x, y$. Thus the set $g$ is furnished with the additive group structure $x, y \rightarrow x+y$, and three multiplicative structures: scalar multiplication $\lambda, x \rightarrow \lambda x$, Lie bracket $x, y \rightarrow[x, y]$, and group multiplication $x, y \rightarrow x y$. 
Given two functions $\phi$ and $\psi$ from a set $X$ to $[0, \infty)$, we write $\phi \lesssim \psi$ if there is a positive constant $M$ such that $\phi(x) \leq M \psi(x)$ for all $x \in X$. If $\phi \lesssim \psi$ and $\psi \lesssim \phi$, we write $\phi \approx \psi$.

\section{Dilations and gauges}

Let $V$ be a finite-dimensional vector space over $R$, and suppose $D: V \rightarrow V$ is a diagonalizable linear transformation with all eigenvalues positive. Define $\delta_{r}=r^{D}$ for $r>0$. Then $\left\{\delta_{r}\right\}$ is a one-parameter group of automorphisms of the additive structure of $V$, and

$$
\lim _{r \rightarrow 0} \delta_{r} x=0, \quad \lim _{r \rightarrow \infty}\left\|\delta_{r} x\right\|=+\infty
$$

(if $x \neq 0$ ). We shall refer to $\left\{\delta_{r}\right\}$ as a group of dilations of $V$.

If $A \subset(0, \infty)$ is the set of eigenvalues of $D$, there is a direct sum decomposition

$$
V=\sum V_{\lambda}, \quad(\lambda \in \Lambda)
$$

where the subspace $V_{\lambda}$ is characterized by

$$
\delta_{r} x=r^{2} x, \quad x \in V_{2} .
$$

Denote by $P_{\lambda}: V \rightarrow V_{\lambda}$ the projection operator, and write $x_{\lambda}=P_{\lambda} x$. Multiplying $D$ by a positive constant if necessary, we shall assume that

$$
\min \Lambda=1 \text {. }
$$

The set $\Lambda$ will be called the spectrum of $\left\{\delta_{r}\right\}$.

It will be useful to introduce growth-measuring functions on $V$ which take into account the different rates of expansion of $\delta_{r}$ in the various $V_{\lambda}$.

Definition. $A \delta_{r}$-homogeneous gauge on $V$ is a continuous function $x \rightarrow$ $|x|$ from $V$ to $[0, \infty)$ such that

(i) $|x|=0 \Leftrightarrow x=0$,

(ii) $\left|\delta_{r} x\right|=r|x|$, all $r>0$.

Fixing the dilation group $\left\{\delta_{r}\right\}$, we shall simply use the term homogeneous gauge.

Example. For any $a>0$, the function

$$
|x|=\left(\sum_{\lambda}\left\|x_{\lambda}\right\|^{a / 2}\right)^{1 / a}
$$

is a homogeneous gauge on $V$. If $a>\max \{\lambda\}$, then it is a $C^{1}$ function on $V \sim\{0\}$.

Lemma 1. If $|\cdot|$ and $|\cdot|_{1}$ are $\delta_{r}$-homogeneous gauges, then $|x| \approx|x|_{1}$. 
Proof. It is enough to consider the case that $|x|$ is given by (3.4) with $a=1$. Set $\phi(x)=|x|_{1} /|x|, x \neq 0$. Then $\phi$ is continuous on $V \sim\{0\}$, and $\phi\left(\delta_{r} x\right)=$ $\phi(x)$. The set $\{|x|=1\}$ is obviously compact, so $C_{1} \leq \phi(x) \leq C_{2}$ if $|x|=1$. Hence $C_{1}|x| \leq|x|_{1} \leq C_{2}|x|$ for all $x$. q.e.d.

By similar compactness arguments, one proves

Lemma 2. If $|\cdot|$ is any homogeneous gauge, then $|-x| \approx|x|,|x+y| \lesssim$ $|x|+|y|$, and $|t x| \lesssim|x|$ uniformly for $0 \leq t \leq 1$.

A function $f$ on $V \sim\{0\}$ will be said to be $\delta_{r}$-homogeneous of degree $\mu$ if

$$
f\left(\delta_{r} x\right)=r^{\mu} f(x)
$$

for all $r>0$ and $x \neq 0$. Fixing a homogeneous gauge, we define

$$
\|f\|_{\infty}=\sup _{|x|=1}|f(x)| \text {. }
$$

Note that $\|f\|_{\infty}<\infty$ if $f$ is continuous, and $|f(x)|<\|f\|_{\infty}|x|^{\mu}$ if $f$ is homogeneous of degree $\mu$.

Let $\left\{x_{i}\right\}$ be a basis for $V$ such that $\delta_{r} x_{i}=r^{\lambda i} x_{i}$, and let $\left\{\xi_{i}\right\}$ be the dilal basis for $V^{*}$. If $f$ is a differentiable function on an open subset of $V$, define

$$
D_{i} f(x)=\left.\frac{d}{d t}\right|_{t=0} f\left(x+t x_{i}\right) .
$$

Suppose now that $f$ is a $C^{1}$ function on $V \sim\{0\}$ which is $\delta_{r}$-homogeneous of degree $\mu$. Then $D_{i} f$ is $\delta_{r}$-homogeneous of degree $\mu-\lambda_{i}$. Define

$$
\|\nabla f\|_{\infty}=\max _{i}\left\|D_{i} f\right\|_{\infty} .
$$

The modulus of continuity of $f$ can then be estimated as follows (cf. [6, Lemma 5.2]).

Lemma 3. Let $x, y \in V \sim\{0\}$ and assume that the straight line $\sigma(t)=$ $(1-t) x+$ ty from $x$ to $y$ does not pass through 0 . Then

$$
|f(x)-f(y)| \leq\|\nabla f\|_{\infty} \sum_{\lambda}\left\|P_{\lambda}(x-y)\right\| \int_{0}^{1}|\sigma(t)|^{\mu-\lambda} d t .
$$

Proof. Since $t \rightarrow f(\sigma(t))$ is $C^{1}$ on $[0,1]$, we can write

$$
f(y)-f(x)=\int_{0}^{1} \frac{d}{d t} f(\sigma(t)) d t .
$$

Now by the chain rule,

$$
\frac{d}{d t} f(\sigma(t))=\sum D_{i} f(\sigma(t)) \xi_{i}(y-x) .
$$


Since $D_{i} f$ is homogeneous of degree $\mu-\lambda_{i}$, we have

$$
\left|D_{i} f(\sigma(t))\right| \leq\|\nabla f\|_{\infty}|\sigma(t)|^{\mu-\lambda_{i}} .
$$

Also $\left|\xi_{i}(z)\right| \leq\left\|P_{\lambda_{i}} z\right\|$. Using these estimates in (3.6) and (3.7), we obtain (3.5).

\section{Filtrations and gradations}

Let $\mathfrak{g}$ be a real Lie algebra, and let $\left\{\mathfrak{g}_{\lambda}\right\}_{\lambda \in R}$ be a family of subspaces of $\mathfrak{g}$ indexed by $\boldsymbol{R}$. It is said to be a real filtration of $g$ if

$$
\mathfrak{g}_{\lambda}=\bigcap_{\mu<\lambda} \mathfrak{g}_{\mu}, \quad\left[\mathfrak{g}_{\lambda}, \mathfrak{g}_{\mu}\right] \subseteq \mathfrak{g}_{\lambda+\mu}
$$

In this paper we assume $\mathfrak{g}$ is nilpotent, and $\left\{\mathfrak{g}_{\lambda}\right\}$ is a real filtration. From (4.1) we see that $\lambda_{1}<\lambda_{2}$ implies $\mathfrak{g}_{\lambda_{1}} \supset \mathfrak{g}_{\lambda_{2}}$, so the family $\left\{\mathfrak{g}_{\lambda}\right\}$ is decreasing. We shall assume also that

$$
\bigcup_{\lambda>0} g_{i}=g, \quad \bigcap_{\lambda>0} g_{i}=0,
$$

so the filtration is positive, exhaustive and separated. If $g_{\lambda}=g_{n}$ when $n-1$ $<\lambda \leq n$ for every positive integer $n$, then the filtration is said to be integral and is determined by the family $\left\{\mathfrak{g}_{n}\right\}_{n \in N}$. Every nilpotent Lie algebra admits an integral filtration satisfying the above conditions (e.g., the descending central series : $\mathfrak{g}_{n+1}=\left[\mathfrak{g}, \mathfrak{g}_{n}\right], \mathfrak{g}_{1}=\mathfrak{g}$ ). Conversely, any finite-dimensional Lie algebra with a filtration satisfying (4.1) and (4.2) is nilpotent.

A special class of filtrations arises from gradations. Namely, suppose $g$ is the vector-space direct sum of subspaces $\left\{\mathfrak{h}_{\lambda}\right\}$, indexed by a finite set $\Lambda \subset(0, \infty)$, which satisfy

$$
\left[\mathfrak{h}_{\lambda}, \mathfrak{h}_{\mu}\right] \subseteq \mathfrak{h}_{\lambda+\mu},
$$

where we set $\mathfrak{h}_{\nu}=0$ if $\nu \notin \Lambda$. Then setting

$$
\mathfrak{g}_{\lambda}=\sum_{\mu \geq \lambda} \mathfrak{h}_{\mu}
$$

defines a positive filtration of $\mathrm{g}$. If $\Lambda \subset[a, b]$, then $\mathfrak{g}_{a}=\mathfrak{g}$ and $\mathfrak{g}_{\lambda}=0$ for $\lambda>b$.

Assume now $\left\{g_{\lambda}\right\}$ is a filtration satisfying (4.1) and (4.2). From it we construct an associated graded Lie algebra gr ( $g$ ) as follows [3, Chapter II, § 4]. Let $\mathfrak{g}_{\lambda}^{+}=\bigcup_{\mu>\lambda} \mathfrak{g}_{\mu}$, and define

$$
\Lambda=\left\{\lambda: \mathfrak{g}_{\lambda}^{+} \neq \mathrm{g}_{\lambda}\right\}
$$

By condition $(4.2), \Lambda \subset(0, \infty)$ and is finite. As a vector space, 


$$
\operatorname{gr}(\mathfrak{g})=\sum \oplus\left(\mathfrak{g}_{\lambda} / \mathfrak{g}_{2}^{+}\right) \quad(\lambda \in \Lambda)
$$

The Lie algebra structure on $\mathrm{gr}(\mathfrak{g})$ is defined by setting

$$
\left[x+\mathfrak{g}_{\lambda}^{+}, y+\mathfrak{g}_{\mu}^{+}\right]=[x, y]+\mathfrak{g}_{\lambda+\mu}^{+}
$$

if $x \in \mathfrak{g}_{2}$ and $y \in \mathfrak{g}_{\mu}$. This is well-defined by virtue of the filtration property (4.1), and $\mathrm{gr}(\mathfrak{g})$ is graded by the subspaces $\left\{\mathfrak{g}_{\lambda} / \mathfrak{g}_{\lambda}^{+}\right\}_{\lambda \in A}$. For any $c>0,\left\{\mathfrak{g}_{c_{\lambda}}\right\}$ satisfies (4.1) and (4.2). Choosing $c$ suitably, we may assume

$$
\min \Lambda=1 \text {. }
$$

We want to compare the Lie algebras $g$ and $g r(\mathfrak{g})$. For this purpose, we choose linear subspaces $\mathfrak{h}_{\lambda} \subset \mathfrak{g}_{\lambda}$ so that

$$
\mathfrak{g}_{\lambda}=\mathfrak{h}_{\lambda} \oplus \mathfrak{g}_{\lambda}^{+}, \quad(\lambda \in \Lambda)
$$

Since the filtration is exhaustive and separating,

$$
\mathfrak{g}=\sum \mathfrak{h}_{\lambda} .
$$

The quotient maps $\mathfrak{h}_{\lambda} \rightarrow \mathfrak{g}_{\lambda} / \mathfrak{g}_{\lambda}^{+}$set up a linear isomorphism between $\mathfrak{g}$ and $\mathrm{gr}(\mathfrak{g})$.

In general, the subspaces $\mathfrak{h}_{\lambda}$ cannot be chosen to satisfy (4.3) (cf. [4], [9]). However, we can use the linear isomorphisms; they define to transfer the Lie bracket from gr $(\mathfrak{g})$ to $\mathfrak{g}$. Denote this new Lie multiplication on $\mathfrak{h}$ by $\{x, y\}$. If $P_{\lambda}: \mathfrak{g} \rightarrow \mathfrak{h}_{\lambda}$ is the projection operator, then $\{x, y\}$ is expressed in terms of the original bracket $[\cdot, \cdot]$ by the formula

$$
\{x, y\}=\sum_{\lambda, \mu} P_{\lambda+\mu}\left[P_{\lambda} x, P_{\mu} y\right] .
$$

Observe that the filtration property (4.1) is equivalent to the equation

$$
P_{\nu}\left[P_{\lambda} x, P_{\mu} y\right]=0, \quad \nu<\lambda+\mu .
$$

Hence $\left\{P_{\lambda} x, P_{\mu} y\right\}$ is obtained by taking the "leading term" of $\left[P_{\lambda} x, P_{\mu} y\right]$.

\section{Comparison of group structures}

Let $\left\{g_{\lambda}\right\}$ be a positive filtration of the nilpotent Lie algebra $\mathfrak{g}$, and fix a choice of subspaces $\left\{\mathfrak{G}_{\lambda}\right\}$ as in $\S 4$. We define a one-parameter group of dilations $\delta_{r}$ on the vector space $g$ by the formula

$$
\delta_{r}=\sum r^{\lambda} P_{\lambda} .
$$

We write $x y$ for the Lie group multiplication on $g$ associated with $[x, y]$, and $x \circ y$ for the Lie group multiplication on $g$ associated with $\{x, y\}$. We thus have three group structures on $\mathrm{g}: x+y, x \circ y$, and $x y$. The dilations $\delta_{r}$ are auto- 
morphisms of the first two structures. Group inversion is given by $x \rightarrow-x$ for all three structures.

Let $|x|$ be a $\delta_{r}$-homogeneous gauge. Our first main result is that the maps $x, y \rightarrow x y$ and $x, y \rightarrow x \circ y$ are "asymptotic at infinity" when measured via the gauge, as follows.

Theorem 1. There is a positive constant $\gamma<1$ so that for any $\varepsilon>0$,

$$
|x y-x \circ y| \leq M_{8}(|x|+|y|)^{r}
$$

in the set $\{|x|+|y| \geq \varepsilon\}$. In particular,

$$
\lim _{|x|+|y| \rightarrow \infty} \frac{|x y-x \circ y|}{|x|+|y|}=0
$$

The proof of Theorem 1 will be given in $\S 6$. Here we draw some consequences from (5.1). Since $x \circ y-\delta_{1 / r}\left(\delta_{r} x \delta_{r} y\right)=\delta_{1 / r}\left(\delta_{r} x \circ \delta_{r} y-\delta_{r} x \delta_{r} y\right)$, we deduce that if $|x|+|y| \geq \varepsilon$, then

$$
\left|x \circ y-\delta_{1 / r}\left(\delta_{r} x \delta_{r} y\right)\right| \leq M_{\bullet} r^{r-1}(|x|+|y|)^{r} .
$$

In particular, since $\gamma<1$, this implies

Corollary 1. $x \circ y=\lim _{r \rightarrow \infty} \delta_{1 / r}\left(\delta_{r} x \delta_{r} y\right)$.

Since $\delta_{r}(x \circ y)=\delta_{r} x \circ \delta_{r} y$, it follows directly from (5.2) that if $r \geq \varepsilon$ and $|x|+|y| \geq \varepsilon$, then there is a constant $C_{\varepsilon}$ so that

$$
\left|\delta_{r}(x y)-\left(\delta_{r} x\right)\left(\delta_{r} y\right)\right| \leq C_{\varepsilon} r(|x|+|y|)^{r} .
$$

This implies

Corollary 2. For fixed $r, \delta_{r}$ is an "asymptotic automorphism":

$$
\lim _{|x|+|y| \rightarrow \infty} \frac{\left|\delta_{r}(x y)-\left(\delta_{r} x\right)\left(\delta_{r} y\right)\right|}{|x|+|y|}=0 .
$$

Our next main result is a comparison of the additive group structure of $\mathfrak{g}$ and the nilpotent group structure of $\mathrm{g}$.

Theorem 2. If $\varepsilon>0$, there is a constant $C_{s}$ so that in the region $|x| \geq$ $\varepsilon(1+|y|)$,

$$
\left\|P_{\lambda}(x y-x-y)\right\| \leq C_{\odot}|x|^{\lambda-1}|y| .
$$

In particular, if $1 / a=\max _{1}\{\lambda\}$, then in this region

$$
|x y-x-y| \leq C_{s}|x|^{1-a}|y|^{a} \text {. }
$$

The proof of Theorem 2 will be given in $\S 6$. Let us draw some consequences from (5.4) and (5.5) at this point. 
Corollary 3. The right translation maps $x \rightarrow x y$ and $x \rightarrow x+y$ are "asymptotic at infinity":

$$
\lim _{|x| \rightarrow \infty} \frac{|x y-(x+y)|}{|x|}=0
$$

Combining the results of Theorems 1 and 2, we can estimate the action of translation by $y$ on homogeneous functions, as follows.

Theorem 3. There are positive numbers $M$ and $C$ with the following property: If $f$ is a $C^{1}$ function on $\mathrm{g} \sim\{0\}$ which is $\delta_{r}$-homogeneous of degree $\mu$, then in the region $|x| \geq M(1+|y|)$,

$$
|f(x y)-f(x)| \leq C\|\nabla f\|_{\infty}|x|^{\mu-1}|y| .
$$

The proof of Theorem 3, assuming the results of Theorems 1 and 2, goes as follows: Consider the line segments $\sigma(t)=(1-t) x+t x y$ and $\sigma_{0}(t)=$ $(1-t) x-t x \circ y$, where $0 \leq t \leq 1$. Using a compactness argument and the fact that $\delta_{r}$ is an automorphism of $x \circ y$, Korányi and Vagi [6, Lemma 5.2] show that there exists an $N>0$ such that for $0 \leq t \leq 1$,

$$
|x| \geq N|y| \Rightarrow \frac{1}{2}|x| \leq\left|\sigma_{0}(t)\right| \leq 2|x|
$$

Since $\sigma(t)-\sigma_{0}(t)=t(x y-x \circ y)$, it follows by Theorem 1 that

$$
|x| \geq(|y|+1) \Rightarrow\left|\sigma(t)-\sigma_{0}(t)\right| \lesssim|x|^{r}
$$

where $0 \leq \gamma<1$. Combining (5.7) and (5.8), we conclude that there exists a constant $M \geq 1$ so that in the region $|x| \geq M(|y|+1)$,

$$
M^{-1}|x| \leq|\sigma(t)| \leq M|x|
$$

Furthermore, in this region

$$
\left\|P_{\lambda}(x y-y)\right\| \leq\left\|P_{\lambda}(x y-x-y)\right\|+\left\|P_{\lambda} y\right\| \lesssim|x|^{\lambda-1}|y|,
$$

by Theorem 2. Using these estimates in Lemma 3, we obtain (5.6).

\section{Proofs of Theorems 1 and 2}

As the preliminary step toward proving Theorems 1 and 2, we shall analyze the contribution of each subspace $\mathfrak{h}_{2}$ to the Campbell-Hausdorff formula. For this purpose, introduce a set $S$ of indeterminants $X_{\lambda}, Y_{\lambda}$, with $\lambda$ ranging over $\Lambda$, and denote by $L(S)$ the free Lie algebra over $S$ (cf. [3, Chapter II] for definitions and notations). We use the generators $X_{\lambda}, Y_{\lambda}$ to define a multigradation on $L(S)$. Namely, if $\alpha, \beta \in N^{4}$, then $L(S)_{\alpha \beta}$ is the subspace spanned 
by all "alternants" (iterated commutators of elements of $S$ ) containing $X_{\lambda}, \alpha(\lambda)$ times and $Y_{\lambda}, \beta(\lambda)$ times. Let $\left\{H_{j}^{\alpha \beta}\right\}$ be a Hall basis for $L(S)_{\alpha \beta}$, with each $H_{j}^{\alpha \beta}$ being an alternant in the $X_{\lambda}$ and $Y_{\lambda}$. The formal power series $\log \left(\exp \sum_{\lambda} X_{\lambda} \exp \sum_{\lambda} Y_{\lambda}\right)$ is a Lie element in the completion of the tensor algebra over $S$. Hence there are rational coefficients $C_{j}^{\alpha \beta}$, with $\alpha, \beta$ nonzero and ranging over $N^{\Lambda}$, such that

$$
\log \left(\exp \left(\sum X_{\lambda}\right) \exp \left(\sum Y_{\lambda}\right)\right)=\sum\left(X_{\lambda}+Y_{\lambda}\right)+\sum C_{j}^{\alpha \beta} H_{j}^{\alpha \beta}
$$

(graded form of the Campbell-Hausdorff formula).

Return now to $\mathfrak{g}=\sum \mathfrak{h}_{\lambda}$. $\mathfrak{g}$ carries two Lie algebra structures: $[x, y]$ and $\{x, y\}$. Let $x=\sum x_{\lambda}, y=\sum y_{\lambda}$, and define $H_{j}^{\alpha \beta}[x ; y]$ by the "substitution" $X_{\lambda} \rightarrow x_{\lambda}, Y_{\lambda} \rightarrow y_{\lambda}$, relative to the original Lie bracket $[\cdot, \cdot]$. Similarly, define $H_{j}^{\alpha \beta}\{x ; y\}$ by "substitution" relative to the Lie bracket $\{\cdot, \cdot \cdot\}$ of $\mathrm{gr}(\mathrm{g})$. It then follows by (6.1) that

$$
x y=x+y+\sum C_{j}^{\alpha \beta} H_{j}^{\alpha \beta}[x ; y],
$$

while

$$
x \circ y=x+y+\sum C_{j}^{\alpha \beta} H_{j}^{\alpha \beta}\{x ; y\} .
$$

(By the nilpotence of $\mathfrak{g}$, the sums in (6.2) and (6.3) are finite.)

To make the estimates as simple as possible, let us assume that the norm on $\mathrm{g}$ has been adjusted so that $\|[x, y]\| \leq\|x\|\|y\|$ and also $\|\{x, y\}\| \leq\|x\|\|y\|$. (This can be achieved by scaling the basis for $g$ used to define $\|x\|$.) With this done, we have the estimate

$$
\left\|H_{j}^{\alpha \beta}[x ; y]\right\| \leq \prod_{\lambda}\left\|x_{\lambda}\right\|^{\alpha(\lambda)}\left\|y_{\lambda}\right\|^{\beta(\lambda)} .
$$

The same estimate holds for $H_{j}^{\alpha \beta}\{x ; y\}$. Define the weight $w(\alpha)$ of a multiindex $\alpha \in N^{\Lambda}$ by $w(\alpha)=\sum \lambda \alpha(\lambda)$. Since $\left\|x_{\lambda}\right\| \leq|x|^{\lambda}$, the above estimate may be written as

$$
\left\|H_{j}^{\alpha \beta}[x ; y]\right\| \leq|x|^{w(\alpha)}|y|^{w(\beta)} .
$$

Similarly,

$$
\left\|H_{j}^{\alpha \beta}\{x ; y\}\right\| \leq|x|^{w(\alpha)}|y|^{w(\beta)} .
$$

Proof of Theorem 1. We start with the observation that formula (4.5), expressing $\{x, y\}$ in terms of $\left[P_{2} x, P_{\mu} y\right]$, implies inductively that the alternants appearing in formulas (6.2) and (6.3) are related by

$$
H_{j}^{\alpha \beta}\{x ; y\}=P_{w(\alpha)+w(\beta)} H_{j}^{\alpha \beta}[x ; y] .
$$

Furthermore, $H_{j}^{\alpha \beta}[x ; y] \in \mathfrak{g}_{w(\alpha)+w(\beta)}$, while $H_{j}^{\alpha \beta}\{x ; y\} \in \mathfrak{h}_{w(\alpha)+w(\beta)}$.

Consider now the problem of estimating $x y-x \circ y$. By formulas (6.2) and 
(6.3), it suffices to estimate the differences $H_{j}^{\alpha \beta}[x ; y]-H_{j}^{\alpha \beta}\{x ; y\}$. Using formula (6.6), we see that it suffices to estimate the projections $P_{\lambda} H_{j}^{\alpha \beta}[x ; y]$ when $\lambda>\lambda_{0}=w(\alpha)+w(\beta)$. By estimate (6.4),

$$
\begin{aligned}
\left|P_{\lambda} H_{j}^{\alpha \beta}[x ; y]\right| & =\left\|P_{\lambda} H_{j}^{\alpha \beta}[x ; y]\right\|^{1 / \lambda} \\
& \leq\left\|H_{j}^{\alpha \beta}[x ; y]\right\|^{1 / \lambda} \leq\left(|x|^{w(\alpha)}|y|^{(\nu,(\beta)}\right)^{1 / \lambda} .
\end{aligned}
$$

By the geometric mean-arithmetic mean inequality, this last quantity is bounded by $(|x|+|y|)^{\alpha_{0} / 2}$. Take $\gamma$ as the largest of the numbers $\lambda_{0} / \lambda$ which occur.

Proof of Theorem 2. By formula (6.2) and Lemma 2, it suffices to estimate $\left\|P_{\lambda} H_{j}^{\alpha \beta}[x ; y]\right\|$. But this is zero if $w(\alpha)+w(\beta)>\lambda$. If $w(\alpha)+w(\beta)$ $\leq \lambda$, then by estimate (6.4),

$$
\begin{aligned}
\left\|P_{2} H_{j}^{\alpha \beta}[x ; y]\right\| & \leq|x|^{w(\alpha)}|y|^{w(\beta)} \\
& \leq\left(|x|^{\alpha-1}|y|\right)\left(|x|^{a+b-\lambda}\right)(|y| /|x|)^{b-1} \\
& \leq C_{\iota}|x|^{2-1}|y|
\end{aligned}
$$

provided $|x| \geq \varepsilon(1+|y|)$, where we have written $a=w(\alpha)$ and $b=w(\beta)$. This proves the first statement of Theorem 2 . The second statement then follows because $\max _{2}\left\|P_{\lambda} x\right\|^{1 / \lambda} \approx|x|$, (Lemma 1).

Remark. In case $[x, y]=\{x, y\}$, only the terms with $w(\alpha)+w(\beta)=\lambda$ occur in the above estimate, and the factor $|x|^{a+b-\lambda}=1$. Hence estimates (5.4) and (5.5) hold in the region $|x| \geq \varepsilon|y|$ in the graded case.

\section{Vector fields with polynomial coefficients}

In this section we construct a differential operator realization of the algebraic calculations of $\S 6$. This version is natural to employ when one starts with the group law in exponential coordinates, and will allow us to compare the theorems in $\S 5$ with related results of Auslander, Brezin, and Sacksteder [1]. The first step is to construct some basic filtered nilpotent Lie algebras of differential operators.

Let $V$ be a real, finite-dimensional vector space, and let $\left\{\delta_{r}\right\}$ be a oneparameter group of dilations of $V$, with spectrum $\Lambda$, as in $\S 3$. Every vector $X \in V$ defines a constant-coefficient vector field $D_{X}$ on $V$ by the formula

$$
D_{X} \phi(v)=\left.\frac{d}{d t}\right|_{t=0} \phi(v+t X),
$$

$\phi \in C^{\infty}(V)$, i.e., $D$ is the right regular representation of $V$ considered as an abelian Lie algeba. Denote this space of vector fields by $\mathscr{D}$.

Let $\mathscr{P}$ be the algebra of polynomial functions on $V$. The dilations $\delta_{r}$ induce a contragredient action $\delta_{r}^{*}$ on $\mathscr{P}$ : 


$$
\delta_{r}^{*} \phi(v)=\phi\left(\delta_{r-1} v\right) .
$$

In particular, the spectrum of $\left\{\delta_{r}^{*}\right\}$ on $\mathscr{P}$ is the semigroup $-N \Lambda$ generated by $-\Lambda$.

Let $\mathscr{L}=\mathscr{P} \mathscr{D}$ be the space of all vector fields on $V$ spanned by the operators $\phi D_{X}$, with $\phi \in \mathscr{P}, X \in V$ (the vector fields with polynomial coefficients). Since $D_{X}(\mathscr{P}) \subseteq \mathscr{P}$, it follows that $\mathscr{L}$ is a Lie subalgebra of the Lie algebra of all $C^{\infty}$ vector fields on $V$. The dilations $\delta_{r}$ canonically define Lie algebra automorphisms $\delta_{r}^{\sharp}$ of $\mathscr{L}$, and it is easy to calculate that

$$
\delta_{r}^{\sharp}\left(\phi D_{X}\right)=\left(\delta_{r}^{*} \phi\right) D_{\delta_{r} X} .
$$

In particular, $\delta_{r}^{\sharp}$ is diagonalizable on $\mathscr{L}$ and has spectrum

$$
\Lambda^{\#}=\left\{\lambda-\sum m_{\mu} \mu: \lambda, \mu \in \Lambda, m_{\mu} \in N\right\} .
$$

For $\mu \in \Lambda^{\#}$, set

$$
\mathfrak{h}_{\mu}=\left\{T \in \mathscr{L}: \delta_{r}^{\sharp} T=r^{\mu} T\right\} .
$$

Then $\mathfrak{h}_{\mu}$ is spanned by the vector fields $\phi D_{X}$, such that $\phi\left(\delta_{r} v\right)=r^{\alpha} \phi(v), \delta_{r} X$ $=r^{\beta} X$, and $\beta-\alpha=\mu$. There is a direct sum decomposition

$$
\mathscr{L}=\sum \mathfrak{h}_{\mu} \quad\left(\mu \in \Lambda^{\sharp}\right) .
$$

Since $\delta_{r}^{\#}$ is a Lie algebra automorphism, this decomposition is a gradation of $\mathscr{L}$. The associated filtration is given by

$$
\mathscr{L}_{\lambda}=\sum_{\mu \geq \lambda} \mathfrak{h}_{\mu}
$$

In particular, if we define

$$
\mathfrak{n}=\sum_{\mu>0} \mathfrak{h}_{\mu},
$$

then $\mathfrak{n}$ is a finite-dimensional nilpotent Lie algebra of vector fields.

Examples. 1. If $\delta_{r} v=r v$ (scalar multiplication), then $\Lambda=\{1\}$. Hence $\mathfrak{n}=\mathscr{D}$, the constant-coefficient vector fields, in this case.

2. If $V=V_{1} \oplus V_{2}$, and $\delta_{r}\left(v_{1}=v_{2}\right)=r v_{1}+r^{2} v_{2}\left(v_{i} \in V_{i}\right)$, then $\Lambda=\{1,2\}$. $\mathfrak{n}=\mathfrak{h}_{1} \oplus \mathfrak{h}_{2}$, with $\mathfrak{h}_{1}$ spanned by operators $D_{X_{1}}$ and $f_{1} D_{X_{2}}$, while $\mathfrak{h}_{2}$ is spanned by operators $D_{X_{2}}$ where $X_{i} \in V_{i}$ and $f_{1} \in V_{1}^{\prime}$. Thus $\mathfrak{n}$ is two-step nilpotent, of dimension $=d_{1}\left(d_{2}+1\right)+d_{2}$ where $d_{i}=\operatorname{dim} V_{i}$. If $d_{2}=1, \mathfrak{n}$ is the $\left(2 d_{1}+1\right)$ dimensional Heisenberg algebra.

Suppose now that $G$ is a simply-connected nilpotent Lie group. Then global "canonical coordinates of the first kind" $\left\{x_{i}\right\}_{i=1}^{d}$ can be found for $G$ such that the group operations are expressed as 


$$
\begin{aligned}
\left(x^{-1}\right)_{i} & =-x_{i}, \\
(x y)_{i} & =x_{i}+y_{i}+f_{i}\left(x_{1}, \cdots, x_{i-1} ; y_{1}, \cdots, y_{i-1}\right),
\end{aligned}
$$

where $f_{i}$ is a polynomial function $\left(f_{i}=0\right.$ when $i=1$ and 2$)$. The map $x \mapsto$ $\left(x_{1}, \cdots, x_{d}\right)$ is an analytic manifold isomorphism from $G$ to $\boldsymbol{R}^{d}$. We use this map to identify $G$ and $g$ with $\boldsymbol{R}^{d}$. If $X \in \mathfrak{g}$, then the straight line $\{t X\}_{t \in R}$ through $X$ is the one-parameter subgroup of $G$ generated by $X$.

Given such a presentation of $G$, we use the procedure in $[1, \S 6]$ to define inductively a group of dilations $\delta_{r}$ on $\boldsymbol{R}^{d}$. Namely, we let $X_{i}=(0, \cdots, 1$, $\cdots, 0)\left(1\right.$ in $i$ th place), and set $\delta_{r} X_{i}=r^{\alpha_{i}} X_{i}$. The exponents $\alpha_{i}$ are positive integers chosen successively so that for $i=3, \cdots, d$,

$$
f_{i}\left(\delta_{r} x ; \delta_{r} y\right)=r^{\alpha_{i}} g_{i}(x ; y)+O\left(r^{\alpha_{i}-1}\right) .
$$

$\left(\alpha_{1}\right.$ and $\alpha_{2}$ are arbitrary. In agreement with the normalization of $\S 3$, we take $\alpha_{1}=1$ and $\alpha_{2} \geq \alpha_{1}$.)

Theorem 4. The subspaces

$$
\mathrm{g}_{n}=\operatorname{span}\left\{X_{i}: \alpha_{i} \geq n\right\}
$$

form a Lie algebra filtration of $\mathrm{g}$. The corresponding graded multiplication is

$$
x \circ y=\lim _{r \rightarrow \infty} \delta_{1 / r}\left(\delta_{r} x \delta_{r} y\right),
$$

and is given in coordinate form by

$$
(x \circ y)_{i}=x_{i}+y_{i}+g_{i}(x ; y),
$$

where $g_{i}$ is defined by (7.7).

Proof. Let $\mathscr{L}$ be the Lie algebra of vector fields with polynomial coefficients on $\boldsymbol{R}^{d}$, and extend $\delta_{r}$ to act as Lie algebra automorphisms $\delta_{r}^{\sharp}$ of $\mathscr{L}$. Let $\left\{\mathscr{L}_{n}\right\}_{n \in \boldsymbol{Z}}$ be the filtration of $\mathscr{L}$ determined by $\delta_{r}^{\#}$. (It is an integral filtration since the $\alpha_{i}$ are integers.)

The Lie algebra $g$ can be faithfully represented in $\mathscr{L}$ via the right regular representation. For $Y \in \mathfrak{g}$, let $y(t)=t Y$ be the one-parameter subgroup of $G$ generated by $Y$. Define the vector field $R(Y)$ by

$$
R(Y) \phi(x)=\left.\frac{d}{d t}\right|_{t=0} \phi(x y(t)), \quad \phi \in C^{\infty}\left(\boldsymbol{R}^{d}\right) .
$$

To calculate $R(Y)$, write $D_{i}=\partial / \partial x_{i}$, and set

$$
c_{i j}(x)=\left.\frac{\partial}{\partial y_{i}} f_{j}(x ; y)\right|_{y=0} .
$$


Then by (7.6),

$$
R\left(X_{i}\right)=D_{i}+\sum_{j>i} c_{i j} D_{j} .
$$

Differentiating (7.7) we find that

$$
c_{i j}\left(\delta_{r} x\right)=r^{\mu} \tilde{c}_{i j}(x)+O\left(r^{\mu-1}\right),
$$

where $\mu=\alpha_{j}-\alpha_{i}$ and

$$
\tilde{c}_{i j}(x)=\left.\frac{\partial}{\partial y_{i}} g_{\jmath}(x ; y)\right|_{y=0} .
$$

By formula (7.3) for the action of $\delta_{r}^{\#}$ we thus have

$$
\delta_{r}^{\#} R\left(X_{i}\right) \equiv r^{\alpha_{i}} R\left(X_{i}\right)
$$

modulo $\mathscr{L}_{\alpha_{i}+1}$. Hence $R\left(X_{i}\right) \in \mathscr{L}_{\alpha_{i}}$ and

$$
\delta_{r}^{\sharp} R\left(X_{i}\right) \equiv R\left(\delta_{r} X_{i}\right)
$$

modulo $\mathscr{L}_{\alpha_{i+1}}$. Note that $\delta_{r}$ maps $g$ onto $g$ but is not necessarily a Lie algebra automorphism, while $\delta_{r}^{\#}$ is a Lie algebra automorphism of the larger algebra $\mathscr{L}$ but may map $R(\mathrm{~g})$ out of $R(\mathrm{~g})$.

To verify the filtration property, observe that since $R$ is a representation, (7.12) implies that

$$
\delta_{r}^{\sharp} R\left(\left[X_{i}, X_{j}\right]\right) \equiv r^{\beta} R\left(\left[X_{i}, X_{j}\right]\right)
$$

$\bmod \mathscr{L}_{\beta+1}$, where $\beta=\alpha_{i}+\alpha_{j}$. Hence by (7.13),

$$
\delta_{r}\left(\left[X_{i}, X_{j}\right]\right)=r^{\beta}\left[X_{i}, X_{j}\right]+O\left(r^{\beta+1}\right),
$$

since $R$ is faithful. (7.14) implies immediately that $\left\{g_{n}\right\}$ is a Lie algebra filtration. The eigenspaces for $\delta_{r}$ furnish complements to $\mathfrak{g}_{n+1}$ in $\mathfrak{g}_{n}$. We map gr (g) linearly onto $g$ using these complements, as in $\S 4$. (7.8) and (7.9) are then consequences of Corollary 1 and (7.7).

Remarks. 1. Let $\{X, Y\}$ be the graded Lie bracket on $\mathfrak{g}$ determined by the dilations $\left\{\delta_{r}\right\}$. Define

$$
\tilde{R}\left(X_{i}\right)=D_{i}+\sum_{j>i} \tilde{c}_{i j} D_{\jmath},
$$

where $\tilde{c}_{i j}$ is the polynomial defined by (7.11). From the calculations just made we see that

$$
\tilde{R}(\{X, Y\})=[\tilde{R}(X), \tilde{R}(Y)], \quad \tilde{R}\left(\delta_{r} X\right)=\delta_{r}^{\sharp} \tilde{R}(X)
$$


$\tilde{R}$ is the right regular representation of $\operatorname{gr}(\mathrm{g})$ on $C^{\infty}(G)$, relative to the multiplication $x \circ y$.

2. Our Theorem 1 is a quantitative version of the statement in $[1, \S 6]$ that " $x \circ y$ is a slight change in the group operation of $G$ ".

\section{References}

[ 1 ] L. Auslander, J. Brezin \& R. Sacksteder, A method in metric Diophantine approximation, J. Differential Geometry 6 (1972) 479-496.

[2] G. Birkhoff, Representability of Lie algebras and Lie groups by matrices, Ann. of Math. 38 (1937) 526-532.

[3] N. Bourbaki, Groupes et algébres de Lie, Hermann, Paris, 1968.

[ 4 ] J. L. Dyer, A nilpotent Lie algebra with nilpotent automorphism group, Bull. Amer. Math. Soc. 76 (1970) 52-56.

[ 5 ] R. W. Johnson, Homogeneous Lie algebras and expanding automorphisms, preprint.

[6] A. Korányi \& S. Vagi, Singular integrals in homogeneous spaces and some problems in classical analysis, Ann. Scuola Norm. Sup. Pisa Cl. Sci. 25 (1971) 575-648.

[ 7 ] A. W. Knapp \& E. M. Stein, Intertwining operators for semisimple Lie groups, Ann. of Math. (2) 93 (1971) 489-578.

RUTGERS UNIVERSITY 